Abstract P3-S5.05 Table 1 Positive tests among the 99 specimens subjected to point of care multiplex PCR using the filmarray device

\begin{tabular}{|c|c|c|c|c|c|c|c|}
\hline \multirow[b]{2}{*}{ Detected organism } & \multicolumn{7}{|c|}{ Specimen type } \\
\hline & $\begin{array}{l}\text { Urine } \\
N=44\end{array}$ & $\begin{array}{l}\text { Urethral } \\
\mathrm{N}=5\end{array}$ & $\begin{array}{l}\text { Cervical } \\
N=7\end{array}$ & $\begin{array}{l}\text { Rectal } \\
N=16\end{array}$ & $\begin{array}{l}\text { Oral } \\
N=20\end{array}$ & $\begin{array}{l}\text { Ulcer } \\
N=7\end{array}$ & Tota \\
\hline Neisseria gonorrhoeae & 4 & 2 & & 2 & 2 & & 10 \\
\hline Chlamydia trachomatis & 7 & 2 & & 3 & & & 12 \\
\hline Treponema pallidum & 1 & & & 1 & 1 & 1 & 4 \\
\hline Trichomonas vaginalis & 1 & & 2 & & & & 3 \\
\hline HSV-1 & 2 & & & & & & 2 \\
\hline HSV-2 & & & & & & & 0 \\
\hline Mycoplasma genitalium & 3 & & & & & & 3 \\
\hline Ureaplasma spp. & 17 & 1 & 3 & 4 & 2 & 1 & 28 \\
\hline Haemophilus ducreyi & & & & & & & 0 \\
\hline
\end{tabular}

HSV-2 (6/6, 100\%), and T vaginalis (5/6, 83\%). The STD Panel detected $T$ pallidum in four samples from patients who were subsequently diagnosed with syphilis by serology. Ureaplasma spp. were detected in 28 of 99 (28\%) and M genitalium in three of $99(3 \%)$ specimens. In patients with chronic dysuria where standard clinical testing failed to reveal a cause, we were able to detect infectious agent in 8 of 14 specimens (two HSV-1, five Ureaplasma spp., one $M$ genitalium) see Abstract P3-S5.05 table 1.

Conclusions Point of care STD testing based on the FilmArray platform is a robust clinical diagnostic tool that has the potential to improve public health by providing sensitive and rapid results. This may improve clinical care for persons suspected of infections with STD organisms.

\section{P3-S5.06 SURGERY IN THE TREATMENT OF SEVERE DYSPAREUNIA CAUSED BY VULVAR VESTIBULITIS SYNDROME-A LONG- TERM FOLLOW-UP STUDY}

doi:10.1136/sextrans-2011-050108.473

J Paavonen, T Päivi, L Unkila-Kallio. Helsinki University Hospital, Helsinki, Finland

Background Vulvar vestibulitis syndrome (VVS) causes severe dyspareunia in young women, but is poorly recognised and managed. We wanted to evaluate safety and effectiveness of posterior vestibulec-tomy in the treatment of VVS.

Methods A cohort study of consecutive patients with refractory VVS treated at the University Hospital Vulva Clinic. The study population consisted of 70 women treated by posterior vestibulectomy during 1995-2007. All operated women were invited to participate in a long-term follow-up study. Patient characteristics, baseline visual analogue scale (VAS) for dyspareunia, and data of the postoperative pe-riod were collected. Of the 70 women invited, 57 attended the follow-up, including face-to-face inter-view, gynaecologicalgynaecological examination with swab-touch test for vestibular tenderness, current VAS score for dyspareunia, and McCoy questionnaire for sexual problems. Main outcome measures were short-term and long term complication rates, dyspareunia by VAS score, vestibular tenderness on examination, sexual problem index, and overall patient satisfaction.

Results Ninety-one per cent of the patients were satisfied with the outcome. VAS for dyspareunia de-creased from a median of 9 to a median of $3(66.7 \%$ decrease) $(p<0.001)$. Posterior vestibular tenderness was absent in 34 patients $(64.2 \%)$. Six (8.6\%) patients developed postoperative bleeding and 11 (15.7\%) patients developed mild wound infection, and 4 (5.7\%) patients developed Bartholin's cysts. Conclusion Posterior vestibulectomy is effective in the treatment of severe VVS and provides long-term patient satisfaction.

\section{P3-S5.07 MULTIDISCIPLINARY CLINIC FOR THE TREATMENT OF HEPATITIS C: AN USEFUL TOOL FOR THE MANAGEMENT OF THE PATIENTS; REVIEW OF THE EXPERIENCE OF A REGIONAL HOSPITAL}

doi:10.1136/sextrans-2011-050108.474

${ }^{1} \mathrm{P}$ Dolcé, ${ }^{2} \mathrm{~S}$ Brin-Clément, ${ }^{1} \mathrm{H}$ Bernatchez, ${ }^{1} \mathrm{P}$ Jutras, ${ }^{1} \mathrm{~F}$ Tourangeau. ${ }^{1} \mathrm{CSSS}$ RimouskiNeigette, Rimouski, Canada; ${ }^{2}$ Université du Québec à Rimouski, Rimouski, Canada

Background CSSSRN is a regional hospital with 240 acute-care beds, with a multidisciplinary clinic for the treatment of hepatitis C. This study aimed to review the clinical data from treated cases of hepatitis $\mathrm{C}$, including the evaluation of the multidisciplinary support needed.

Methods All files from patients evaluated for hepatitis C at CSSSRN between January 2000 and March 2009 were reviewed for clinical and microbiological data. Also, the numbers of visits to the different clinics were recorded. The data were analysed with Epi Info 3.5.1.

Results A total of 165 cases of hepatitis C were evaluated at our facility and 46 treatments were initiated in 42 patients (four patients had two treatments). The average age was 41 years old (range 22-58) and 78\% were men. All, except one, were native from the province of Quebec. The acquisition of hepatitis $C$ was: injection or inhalation drug use (74\%), blood transfusion (19\%) and other causes $(7 \%)$. The majority of treated individuals were heterosexual (93\%), 55\% had a history of alcohol abuse, 30\% have been in detention, 19\% had attempted suicide and 5\% had done prostitution. HIV coinfection was present in three patients and none had HBV coinfection. The genotypes observed were 1 (55\%), 3 (29\%), 2 $(9 \%)$ and 4 (7\%). Liver biopsy was performed in 26 patients, eight had cirrhosis and one developed liver carcinoma. The treatments received were standard interferon alfa- $2 \mathrm{~b} /$ ribavirine (seven cases, 2000-2002), peginterferon alfa-2b/ribavirine (22 cases, 2003-2006), and peginterferon alfa-2a/ribavirine (17 cases, 2005-2008). Treatment was completed in $78 \%$ of patients. Sustained virological response (SVR) was achieved in 53\% of genotypes 1 and 4; and in $69 \%$ of genotypes 2 and 3. During treatment, an average of 30 visits per patient was recorded. The frequency and mean number of visits were: clinical nurses from the ambulatory care unit $(100 \%, 14)$, infectious diseases $(100 \%, 10)$, gastroenterology $(100 \%, 5)$, psychiatry (35\%) and dietetic (16\%).

Conclusion A SVR was achieved in 53\% (genotypes 1 and 4) and $69 \%$ (genotypes 2 and 3 ) of patients. The treatment of hepatitis $C$ involved an average of 30 visits, with many actors, which is eased by a multidisciplinary clinic.

\section{Clinical sciences poster session 6: syphilis P3-S6.01 VALIDATION OF A TREPONEMIC ANTIBODIES QUIMIOLUMINISCENCE AUTOMATED TEST FOR SYPHILIS DETECTION AMONG INMATES OF MEXICO CITY}

doi:10.1136/sextrans-2011-050108.475

${ }^{1} \mathrm{~L}$ Juárez-Figueroa, ${ }^{2} \mathrm{P}$ Iracheta, ${ }^{3} \mathrm{C}$ Conde-Glez, ${ }^{3} \mathrm{~S}$ Bautista-Arredondo, ${ }^{2} \mathrm{~A}$ GonzálezRodriguez. ${ }^{1}$ HIV/STI Program of Mexico City, Mexico; ${ }^{2}$ HIVIAIDS Program of Mexico City, Mexico; ${ }^{3}$ National Institute of Public Health, Mexico

Background When surveying large groups at risk for STI, the search of anti $T$ pallidum $\mathrm{Ab}$ is a valuable tool both for assessing risks associated with syphilis acquisition and, if followed by VDRL test, also for syphilis cases detection. By the other hand testing first with VDRL a large number of samples followed by confirmation with anti TP specific test would be laborious and prone to errors while the data of past cured syphilis would be lost. On 2010 the HIV/AIDS Program of Mexico City and the National Institute of Public Health, Mexico initiated a health survey of around 40000 inmates of the city for assessing syphilis and 
other STIs. Presently more than 18000 inmates have been surveyed for HIV, HBV HVC and syphilis using the automated immunoquimioluminiscence analysis system Abbott Architect i2000. The accuracy of Abbott Architect Syphilis TP (ASTP) for detecting treated and untreated syphilis was reported before thus our study focused in validate ASTP as compared with an accepted treponemic test.

Methods For evaluatig the sensitivity and specificity of ASTP in the context of our HIV clinic we compared ASTP with a test extensively used for syphilis confirmation, Treponema pallidum haemagglutination assay (TPHA). Samples were assayed with ASTP in pools of four sera and each positive pool developed and re-assayed with ASTP to find one or more individual positive samples which were further assayed with tittered VDRL. ASTP- pools were not re-assayed and individual samples were scored as negative for syphilis. We selected 218 ASTP+ and 1920 ASTP - individual consecutive samples to be tested with BioRad Syphilis TPHA.

Results From 218 ASTP+ samples 212 were TPHA+ and all 1920 ASTP - were also TPHA-. ASTP and TPHA detected all 77 VDRL+ samples thus considered diagnostic of latent or active syphilis. Six ASTP+/TPHA - samples and 135 ASTP-/TPHA- were also VDRL - and considered as evidence of treated/cured syphilis. Using TPHA as gold standard ASTP Sensitivity was $100 \%$ and Specificity was $99.7 \%$. In two ASTP- pools that showed more than 0.6 but $<1$ S/CO reading, a sample weakly positive by ASTP and TPHA but VDRL - was found when assayed individually.

Conclusions Reverting the traditional algorithm of syphilis diagnosis by first determining TP specific antibodies with ASTP followed by tittered VDRL of positive samples is highly accurate even if done in pools of four sera. This approach allows also the identification of epidemiologically valuable data of cured syphilis.

\section{P3-S6.02 IS FOURFOLD DROP OF THE NONTREPONEMAL ANTIBODY TITRESTITRES AT THREE OR 6 MONTHS AFTER EARLY SYPHILIS TREATMENT AN EFFECTIVENESS "CRITERION?"}

doi:10.1136/sextrans-2011-050108.476

P Zhou, X Gu, H Lu, Z Guan, Y Oian. Shanghai Skin Disease Hospital, Shanghai, China

Background At present the criteria for treatment effective in early syphilis is the disappearance of clinical symptoms and signs and four-fold decrease of nontreponemal antibody titres within 3 to 6 months after therapy. However, how to evaluate if syphilis is cured after treatment remains difficult and controversial.

Methods Secondary syphilis patients whose rapid plasma reagin (RPR) titres did not turn negative at least 24 months after treatment were enrolled in this study and their CSF were evaluated. The criteria for enrolment were: (1) RPR titres declined fourfold within 3 months after therapy for secondary syphilis; (2) patients denied high risk sexual behaviour following syphilis treatment; (3) RPR titre did not turn negative at least 24 months after treatment; and (4) HIV negative. The criteria for neurosyphilis were: (1) CSF leukocyte count was elevated, and/or (2) proteins were abnormal, and (3) a reactive VDRL-CSF test in the absence of substantial contamination of CSF with blood, and (4) a reactive TPPA-CSF test, and (5) a negative HIV test, and (6) with or without neurological manifestations, and (7) excluding other possible CNS infections.

Results There were 14 male and three female patients who met the criteria for neurosyphilis. The CSF leukocyte count was elevated in 10 patients among which nine also had CSF proteins elevated. The other three patients had CSF proteins elevated only. CSF-VDRL and CSF-TPPA were reactive in all 17 patients. There were four cases presenting notable neurological and psychiatric manifestations, and other 13 had no signs and symptoms of CNS when they entered the study. The clinical symptoms and signs disappeared or improved in
Abstract P3-S6.02 Table 1 RPR and CSF before anti-neurosyphilis treatment

\begin{tabular}{|c|c|c|c|c|c|c|}
\hline No. & $\begin{array}{l}\text { Initial } \\
\text { RPR }\end{array}$ & $\begin{array}{l}\text { RPR } \\
\text { (3 months after } \\
\left.\text { treatment }{ }^{*}\right)\end{array}$ & $\begin{array}{l}\text { RPR } \\
\text { (24 months } \\
\text { after treatment*) }\end{array}$ & $\begin{array}{l}\text { CSF/WB } \\
(0-8) \times 10^{6} / 1\end{array}$ & $\begin{array}{l}\text { CSF/protein } \\
(150-450) \\
\mathrm{mg} / \mathrm{l}\end{array}$ & $\begin{array}{l}\text { CSF/ } \\
\text { VDRL }\end{array}$ \\
\hline 1 & $1: 64$ & $1: 8$ & $1: 16$ & $8.1 \uparrow$ & $860 \uparrow$ & $1: 4$ \\
\hline 2 & $1: 16$ & $1: 4$ & $1: 4$ & 3 & 441 & $1: 1$ \\
\hline 3 & $1: 32$ & $1: 8$ & $1: 8$ & 1.1 & $890 \uparrow$ & $1: 2$ \\
\hline 4 & $1: 64$ & $1: 6$ & $1: 32$ & $300 \uparrow$ & $1860 \uparrow$ & $1: 8$ \\
\hline 5 & $1: 32$ & $1: 4$ & $1: 8$ & 3.8 & $1050 \uparrow$ & $1: 4$ \\
\hline 6 & 1:128 & $1: 32$ & $1: 64$ & $8.2 \uparrow$ & $650 \uparrow$ & 1:16 \\
\hline 7 & $1: 64$ & $1: 16$ & $1: 16$ & $9.8 \uparrow$ & $460 \uparrow$ & $1: 4$ \\
\hline 8 & $1: 256$ & $1: 32$ & $1: 32$ & 5.8 & $580 \uparrow$ & $1: 8$ \\
\hline 9 & $1: 64$ & $1: 8$ & $1: 8$ & $26 \uparrow$ & $460 \uparrow$ & $1: 4$ \\
\hline 10 & 1:128 & $1: 32$ & $1: 32$ & $8.8 \uparrow$ & 280 & $1: 8$ \\
\hline 11 & $1: 16$ & $1: 4$ & $1: 8$ & $9 \uparrow$ & $540 \uparrow$ & $1: 8$ \\
\hline 12 & 1:128 & $1: 16$ & $1: 16$ & $17 \uparrow$ & $570 \uparrow$ & $1: 4$ \\
\hline 13 & $1: 256$ & $1: 64$ & $1: 64$ & $38 \uparrow$ & $590 \uparrow$ & 1:16 \\
\hline 14 & 1:128 & $1: 32$ & $1: 8$ & 2.2 & 430 & $1: 2$ \\
\hline 15 & $1: 256$ & $1: 32$ & $1: 16$ & $18.8 \uparrow$ & $800 \uparrow$ & $1: 4$ \\
\hline 16 & $1: 64$ & $1: 16$ & $1: 8$ & 3.3 & 430 & $1: 1$ \\
\hline 17 & $1: 16$ & $1: 4$ & $1: 4$ & 3 & 280 & $1: 1$ \\
\hline
\end{tabular}

${ }^{*}$ Anti-secondary syphilis treatment.

four patients, and CSF-WBC in those nine patients turned to normal after treatment. CSF-protein declined accordingly but did not turn to normal in four cases see Abstract P3-S6.02 table 1.

Conclusions A four-fold decrease in serological titres and resolution of lesions of early syphilis may not predict success. The occurrence of failure after standard therapies suggests that the current criteria for "treatment effective" are questionable. There is a need of continuing to evaluate early syphilis patients who meet the criteria for "treatment effective" and whose nontreponemal antibody titres fail to turn negative afterwards.

\section{P3-S6.03 SEROREVERSION OF TREPONEMAL TESTS IN CASES MEETING CANADIAN SURVEILLANCE CRITERIA FOR CONFIRMED CONGENITAL SYPHILIS}

doi:10.1136/sextrans-2011-050108.477

${ }^{1} \mathrm{~S}$ Ameeta, ${ }^{1} \mathrm{~T}$ Guenette, ${ }^{1} \mathrm{~J}$ Bergman, ${ }^{1} \mathrm{~J}$ Gratrix, ${ }^{1} \mathrm{P}$ Parker, ${ }^{1} \mathrm{~B}$ Anderson, ${ }^{2} \mathrm{~S}$ Plitt, ${ }^{3} \mathrm{~B}$ Lee, ${ }^{3} \mathrm{~J}$ Robinson. ${ }^{1}$ Alberta Health Services, Edmonton STI Clinic, Edmonton, Canada; ${ }^{2}$ Public Health Agency of Canada, Canada; ${ }^{3}$ University of Alberta, Canada

Background Serologic tests for syphilis remain the mainstay of diagnosis. However, diagnosis of congenital syphilis is complicated by the passive transfer of maternal antibodies to the infant. Non treponemal test (NTT) titres should decline by age 3 months and should be non reactive by age 6 months if the infant was not infected or was infected but adequately treated. Limited data exist on the serologic outcome of treponemal tests (TT) in cases with clinical or laboratory evidence of congenital syphilis at birth.

Methods Cases meeting Canadian surveillance criteria for confirmed early congenital syphilis [within 2 years of birth] (http://www. phac-aspc.gc.ca/publicat/ccdr-rmtc/09vol35/35s2/Syphilis-eng.php) were reviewed from the Alberta Health Services Edmonton zone from 2005 to 2010. Under Alberta's Public Health Act, maternal stage, treatment information and serologic follow-up and infant clinical, laboratory and treatment information are obtained and stored in a provincial STI database.

Results 22 cases met surveillance criteria for confirmed congenital syphilis: six were either stillborn/deceased at birth, three are still 